\title{
Local regulation of postprandial motor responses in ileal pouches
}

\author{
A Mularczyk, E Contessini-Avesani, B Cesana, P A Bianchi, G Basilisco
}

\begin{abstract}
Background-Local mechanisms are involved in the postprandial regulation of ileal tone in healthy subjects, but whether these mechanisms affect the postprandial tonic response of ileal pouches has not yet been investigated.

Aims-To study the effect of a meal on pouch tone and phasic motor activity in patients with gut continuity or ileostomy and, in the latter group, the effect of a pouch perfusion with chyme or saline. Patients-Twenty patients with ileal pouches: 10 with gut continuity and 10 with ileostomy.

Methods-Pouch tone and the frequency of phasic volume events were recorded with a barostat under fasting and postprandial conditions and after perfusion of the isolated pouch with chyme or saline.

Results-The meal increased pouch tone and the frequency of phasic volume events in the patients with gut continuity, but not in those with ileostomy. Pouch perfusion with chyme induced a greater increase in pouch tone than saline.

Conclusions-The meal stimulated pouch tone and phasic motor activity. These effects were at least partially related to local pouch stimulation by intraluminal contents.

(Gut 1999;45:575-580)
\end{abstract}

Gastroenterologia, Istituto di Scienze

Mediche, Università

degli Studi di Milano, Milan, Italy

A Mularczyk

P A Bianchi

G Basilisco

Cattedra di Chirurgia dell'Apparato

Digerente dell'

Università degli Studi

di Milano, Milan, Italy

E Contessini-Avesani

Laboratorio

Epidemiologico,

IRCCS-Ospedale

Maggiore di Milano,

Milan, Italy

B Cesana

Correspondence to: Dr G Basilisco, Department of Gastroenterology, Padiglione Granelli, Via Sforza 35, 20122 Milan, Italy.

Accepted for publication 14 April 1999
Keywords: ileal pouches; postprandial motor responses; ileal tone; ileostomy; motor activity; barostat

Since Parks and colleagues ${ }^{1}$ published the first description of restorative proctocolectomy with ileal pouch-anal anastomosis in 1980, this technique has become the treatment of choice in most patients with ulcerative colitis and familial adenomatous polyposis. Although the reservoir works satisfactorily in most cases, some patients have a high stool frequency and incontinence, ${ }^{2}$ and a large number of studies have tried to find a relation between these clinical problems and pouch function. Stool frequency has been shown to be inversely correlated with pouch volume, ${ }^{3-5}$ with distension of the pouch to a volume of about $50 \%$ of the maximum tolerated volume triggering the pouch contractions (known as high amplitude pressure waves) associated with defecation. ${ }^{5-7}$ However, although these studies have documented the relevance of pouch volume on stool frequency, the factors that can dynamically influence pouch volume have not yet been investigated.
Recent electronic barostat studies of healthy subjects have shown that a meal induces an immediate increase in the tone and phasic motor activity of the terminal ileum followed by prolonged relaxation ${ }^{8}$; these motor responses are at least partially regulated by the local stimulation of chemoreceptors by nutrients. ${ }^{9}{ }^{10}$ Moreover, in patients with ileal pouches, the phasic motor activity of the pouch has been found to increase in response to an intraduodenal lipid infusion; as this increase is not observed in the pouches of patients whose ileal contents are diverted through an ileostomy, the motor response induced by lipids may be related to the arrival of intraluminal contents in the pouch. ${ }^{11}$ These observations suggest that the consumption of a meal may increase pouch tone-that is, reduce pouch volume and increase the frequency of high amplitude pressure waves. Moreover, this meal induced increase in the tonic and phasic motor activity of the pouch may be at least partially regulated by local mechanisms. To explore this hypothesis, we verified the effect of a meal on pouch tone and phasic motor activity in patients with gut continuity or isolated pouches. Furthermore, in the case of isolated pouches, we compared the effects of local perfusions of identical volumes of chyme and saline on pouch motor activity. An electronic barostat ${ }^{12}$ was used to record pouch motor activity, as this technique seemed to be more convenient than manometry for assessing tonic and phasic motor activity in a large capacity organ. ${ }^{13}$

\section{Patients and methods}

We studied 20 patients with ileal pouch-anal anastomosis (10 men and 10 women, with a mean (SD) age of 37 (12) years, range 18-64). Ten had undergone ileal pouch-anal anastomosis 37 (6) months previously (range 16-66), and the other 10 had undergone ileal pouchanal anastomosis and temporary ileostomy 13 (2) weeks previously, with a small piece of the intestinal wall being left intact to connect the two loops of the ileostomy and maintain the continuity of the enteric nervous system. Sixteen patients were operated on for ulcerative colitis and four for familial adenomatous polyposis; all of them were given a hand sewn endoanal anastomosis. All of the pouches were S-shaped. None of the patients experienced any short term postoperative, sexual, or urinary complications.

The daily stool frequency in the patients with gut continuity, which was assessed retrospectively at the time of the study, was $3.4(0.7)$, with most of the bowel movements $(2.2(0.6))$ 
occurring during the first postprandial hour. None of these patients had to intubate the pouch for evacuation. The discrimination of flatus from stools was good in only two patients. Half of them reported diurnal and nocturnal incontinence. Only one patient used loperamide occasionally, which was stopped 10 days before the study. Pouchitis was excluded on the basis of clinical, endoscopic, and histological data; the pouchitis disease activity index ${ }^{14}$ was less than 2 (range $0-2$ ) in all of the patients during the month preceding the study.

All of the patients gave their written informed consent to the study after the protocol had been explained to them, which was prepared in accordance with the Declaration of Helsinki and approved by the local ethics committee.

The electronic barostat used to record pouch tone consists of a pressure transducer linked through an electronic feedback mechanism to an air injection-aspiration system driven by a computer (Synectics Visceral Stimulator; Medtronic Synectics Medical, Milano, Italy). The air flow rate was $38 \mathrm{ml} / \mathrm{s}$. The barostat's internal compliance was $0.5 \mathrm{ml} / \mathrm{mm} \mathrm{Hg}$ and was linear in the range of pressures from 0 to 55 $\mathrm{mm} \mathrm{Hg}$. Air compressibility was taken into account by calculating the volume as "volume without correction-compression factor $x$ measured pressure". The default compression factor was $0.5 \mathrm{ml} / \mathrm{mm} \mathrm{Hg}$. The intrabag pressure and bag volume were recorded on a computer using version 1.11 of the Polygram for Windows program. The barostat was equipped with a safety system that blocked any procedure if the intrabag pressure exceeded 55 $\mathrm{mm} \mathrm{Hg}$ for five seconds.

A thin walled plastic bag (Mobile Chemical Company, Pittsford, New York, USA) was tied 0.5 and $8.5 \mathrm{~cm}$ from the distal end of a doublelumen polyvinyl tube (Salem Sump Tube; Sherwood Medical, Petit Rechain, Belgium; outer diameter $4.7 \mathrm{~mm}$ ). One lumen of the tube was attached to the pressure sensor and the other to the air injection-aspiration system of the barostat. Preliminary testing at room temperature showed that the bag did not contribute to resistance to inflation within the volume range $0-300 \mathrm{ml}$. At $300 \mathrm{ml}$ (radius 4.6 $\mathrm{cm}$ ), the intrabag pressure was less than $1 \mathrm{~mm}$ $\mathrm{Hg}$. The maximum volume of the bag was 353 (27) $\mathrm{ml}$ (range $310-400 \mathrm{ml}$ ).

\section{STUDY DESIGN}

All of the subjects followed a low fibre diet for 48 hours, and were then studied after an overnight fast. The pouch was cleaned using a 500 $\mathrm{ml}$ tap water enema four hours before the study. All of the procedures were performed with the subjects lying in the right lateral position; the patients were not allowed to change body position during the recording periods. The barostat bag assembly was checked for air leakage at a pressure of $15 \mathrm{~mm} \mathrm{Hg}$ for $10 \mathrm{~min}$ utes before the study. The deflated bag was placed in the pouch with the caudal end $6 \mathrm{~cm}$ from the anal verge. The patients were asked to report the sensations experienced (desire to defecate, urgency, and discomfort), by pressing a button corresponding to the sensation during the recording periods. If discomfort was felt, the procedure was interrupted.

\section{Postprandial motor responses}

To record pouch tone and phasic volume events, the operating pressure was individually selected for each patient and then kept constant throughout the study. This pressure was $2 \mathrm{~mm} \mathrm{Hg}$ higher than the pressure at which respiratory movements could be identified; moreover, the bag volume had to be greater than $50 \mathrm{ml}$ at the selected operating pressure. At the selected pressure, none of the patients perceived a desire to defecate. Pouch tone and phasic volume events were recorded for one hour under fasting conditions and for one hour after a $4.18 \mathrm{MJ}$ liquid meal (250 ml: $20 \%$ lipids, $30 \%$ proteins, and $50 \%$ carbohydrates) consumed by all the patients through a straw over five minutes.

\section{Pouch distensibility}

To investigate differences in fasting volumes between isolated pouches and those in continuity with the gut, pouch distensibility was measured in separate experiments in six fasting patients with gut continuity and in 10 with ileostomy. The foot of the bed was elevated to $30^{\circ}$ to minimise the gravitational effects of the abdominal viscera ${ }^{15}$ on the pouch. After an initial distension at a rate of $100 \mathrm{ml} / \mathrm{min}$ for three minutes and a five minute rest period, one volume-controlled distension was performed by inflating the bag at a rate of $100 \mathrm{ml} / \mathrm{min}$ for three minutes. After bag deflation and a five minute rest, one stepwise pressure-controlled distension was performed at pressures of 1,3 , $5,7,9,11,13,15,20,25,30$, and $35 \mathrm{~mm} \mathrm{Hg}$, each lasting for one minute.

\section{Ileostomy output}

The ileostomy output was collected from six patients with isolated pouches during the 15 minutes before and at 15 minute intervals for one hour after the meal. The ileostomy output collected during the 30-60 minute interval (chyme) was used in the following experiments of pouch perfusion.

\section{Perfusions of isolated pouches}

These studies were performed to assess the effect of chyme in comparison with saline on pouch motor responses. Six patients with isolated pouches were studied. The operating pressure was selected as previously described. Moreover, a Foley catheter (14 Ch; Bard Limited, Crawley, West Sussex, UK) was inserted through the ileostomy into the limb in continuity with the pouch for $5 \mathrm{~cm}$, and its balloon inflated with $10 \mathrm{ml}$ of air. After a 15 minute rest and a five minute baseline recording, the pouch was perfused through the Foley catheter. A 40 $\mathrm{ml}$ volume of saline or chyme was infused into each subject over four minutes through a syringe in a random order and on different days. Pouch tone and phasic volume events were recorded during the 30 minutes after the perfusions. 


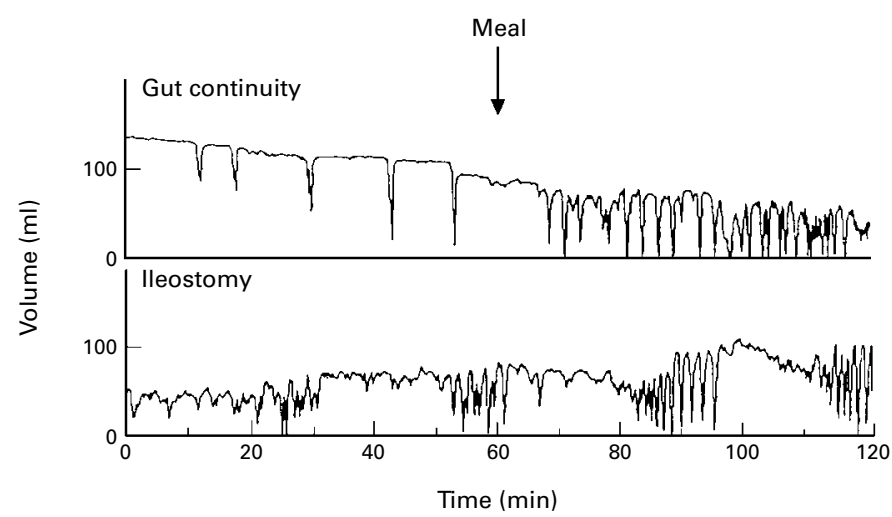

Figure 1 Representative tracings showing the effect of a meal on pouch tone and the number of phasic volume events in one patient with gut continuity and one with ileostomy. The distending pressure was $7 \mathrm{~mm} \mathrm{Hg}$ throughout the experiments in both cases. Note the progressive decrease in pouch volume and the increased frequency of rapid volume changes after the meal only in the pouch in continuity with the gut.

\section{DATA MANAGEMENT}

Recording under isobaric conditions

The changes in bag volume recorded under isobaric conditions were taken to reflect changes in pouch tone, with a decrease in pouch volume representing an increase in pouch tone and vice versa. Rapid changes in bag volume of more than $10 \%$ of the baseline volume lasting more than five seconds and less than two minutes were considered as phasic volume events. ${ }^{13}$ Bag volumes were calculated by the computer. Phasic volume events were counted visually and excluded from the calculation of the mean bag volume.

\section{Pouch distensibility}

The absolute pressure values over atmospheric pressure during the volume-controlled distensions were analysed at each $10 \mathrm{ml}$ increment. The volumes during the pressure-controlled distensions were calculated by measuring the mean volume during the last 10 seconds of distension at each pressure level, during which the volume variations were always less than $10 \mathrm{ml}$. Two pouch distensibility indices were calculated: the pressure/volume ratio at the maximum distension volume tolerated by all of the patients during volume-controlled distension $(90 \mathrm{ml})$, and the slope of the volume/pressure linear relation in the range of pressures tolerated by all of the patients during pressurecontrolled distension (1-11 mm Hg).

\section{Statistical analysis}

The data are given as mean (SD). The statistical analysis was performed using mixed factorial analysis of variance for repeated measurements. ${ }^{16}$ Natural logarithm transformed values were used in the analysis to achieve a Gaussian distribution of the variables investigated. The pattern of volume changes over time was assessed by orthogonal polynomials. ${ }^{17}$ The significance level of the within-patient terms was corrected as described by Huynh and Feldt. ${ }^{18}$ For the perfusion studies of the isolated pouches, analysis of variance was carried out considering baseline and each of the subsequent times; the significance level of the interaction "chyme or

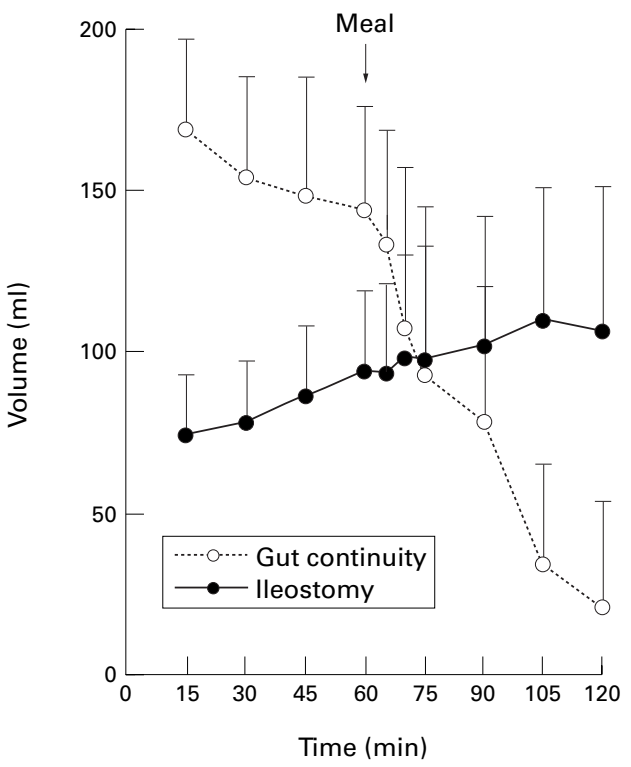

Figure 2 Effect of a meal on pouch tone in patients with gut continuity or ileostomy. Under fasting conditions, pouch volumes were significantly greater in the patients with gut continuity than in those with ileostomy $(p=0.0001)$. The meal induced a significant decrease in pouch volume in the patients with gut continuity ( $p=0.0014$ ), but not in those with ileostomy. The distending pressure was constant throughout the experiments. Results are mean (SD).

saline by time" was therefore established at 0.0125 according to Bonferroni's correction. The Mann-Whitney $U$ test was used to compare the distensibility indexes, Fisher's exact test was used to compare the frequency data, and Pearson's correlation test to investigate correlations between variables.

\section{Results}

POSTPRANDIAL MOTOR RESPONSES

Figure 1 shows representative tracings from one patient with gut continuity and one with ileostomy. Pouch tone and the frequency of phasic volume events increased after the meal in the patient with gut continuity, but not in the patient with ileostomy.

The intrabag operating pressures were similar in the patients with gut continuity and in those with ileostomy (6.4 (1.4) and 7.7 (2.5) $\mathrm{mm} \mathrm{Hg}$ respectively; $\mathrm{p}=\mathrm{NS}$ ). Figure 2 shows the pouch volumes in the two groups of patients under fasting and postprandial conditions. Under fasting conditions, the pouch volumes for the patients with gut continuity were significantly different from those for the patients with ileostomy ( $p=0.0001)$; furthermore, the pouch volumes linearly decreased in the former $(p=0.0118)$ and linearly increased $(\mathrm{p}=0.0002)$ in the latter. In the patients with gut continuity, meal consumption was followed by a significant decrease in volume ( $p=$ 0.0014), which continued up to the end of the study following a linear function $(p=0.0034)$; in the patients with ileostomy, pouch volume was not significantly affected by meal ingestion.

No between-group difference was observed in the frequency of phasic volume events under fasting conditions (fig 3), but the frequency 


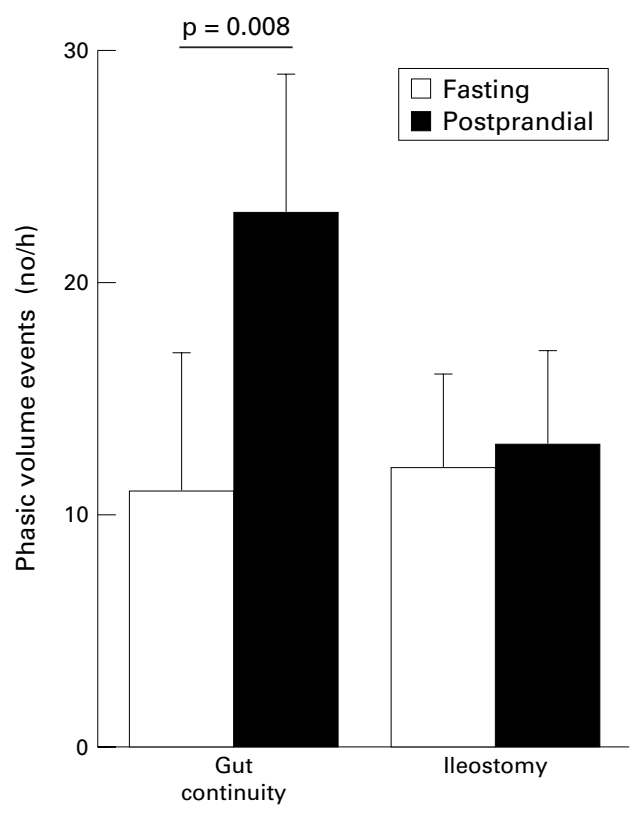

Figure 3 Effect of a meal on the number of phasic volume events in patients with gut continuity or ileostomy. The mean ( $S D$ ) of phasic volume events in the hour preceding and following the meal are shown for the two groups of patients. The number of phasic volume events after the meal significantly increased only in the patients with gut continuity.

increased significantly $(\mathrm{p}=0.008)$ during the postprandial period in the patients with gut continuity but not in those with ileostomy (fig 3).

Three of the patients with gut continuity reported a desire to defecate in the postprandial period, and eight evacuated chyme after the removal of the barostat bag.

Mean pouch volume under fasting conditions tended to be inversely correlated with the daily stool frequency reported by the patients with gut continuity (Pearson's correlation coefficient $=-0.53 ; \mathrm{p}=0.17)$. The frequency of phasic volume events under fasting conditions, and postprandial changes (mean values

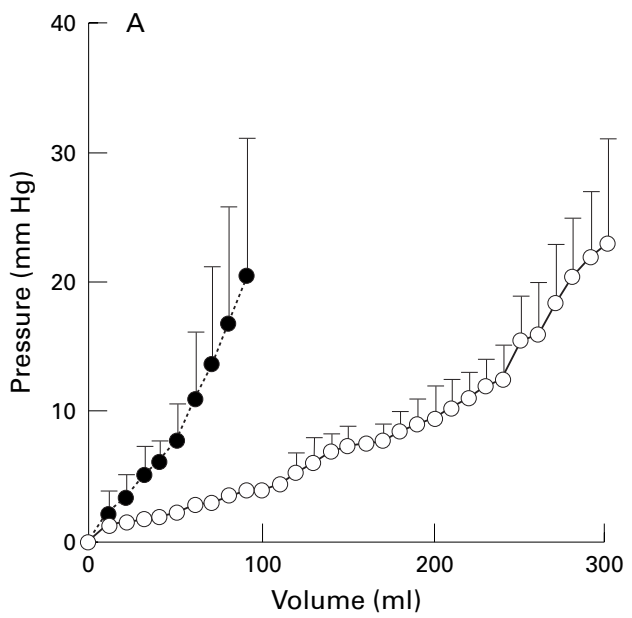

in the postprandial hour - mean values under fasting conditions) in pouch volume and the frequency of phasic volume events did not correlate with daily stool frequency.

POUCH DISTENSIBILITY

Figure 4 shows pouch distensibility during the volume- and pressure-controlled distensions. The distensibility of the isolated pouches was less than that of the pouches in continuity with the gut. The pressure/volume ratio during volume-controlled distension was significantly lower in patients with gut continuity than in those with ileostomy $(0.0423 \quad(0.002) \mathrm{mm}$ $\mathrm{Hg} / \mathrm{ml} v 0.227(0.117) \mathrm{mm} \mathrm{Hg} / \mathrm{ml}$ respectively; $\mathrm{p}=0.0001)$, and the slope of the volume/ pressure relation during pressure-controlled distension was significantly steeper in the former than in the latter $(15.5$ (3.3) $\mathrm{ml} / \mathrm{mm} \mathrm{Hg}$ v $6.1(1.9) \mathrm{ml} / \mathrm{mm} \mathrm{Hg} ; \mathrm{p}=0.0001)$.

None of the patients with gut continuity reported a desire to defecate, urgency, or interrupted the procedure because of discomfort, whereas all of the patients with ileostomy interrupted the distensions because of discomfort $(\mathrm{p}=0.001)$; a desire to defecate and urgency preceded the experience of discomfort in respectively eight and seven of the 10 patients.

ILEOSTOMY OUTPUT

The ileostomy output in the patients with isolated pouches was erratic in the minutes before and after the meal, and mainly characterised by solid residues and fluids in the first 15-30 minutes after the meal; chyme was clearly detectable in the 30-60 minute period. Ileostomy output in the $-15-0,0-15,15-30$, 30-45, and 45-60 minute periods was 3 (4), 5 (8), 32 (36), 59 (54), and 105 (79) $\mathrm{ml}$ respectively.

PERFUSIONS OF ISOLATED POUCHES

A decrease in pouch volume was observed in the five minute period after chyme perfusion, whereas an equal volume of saline did not

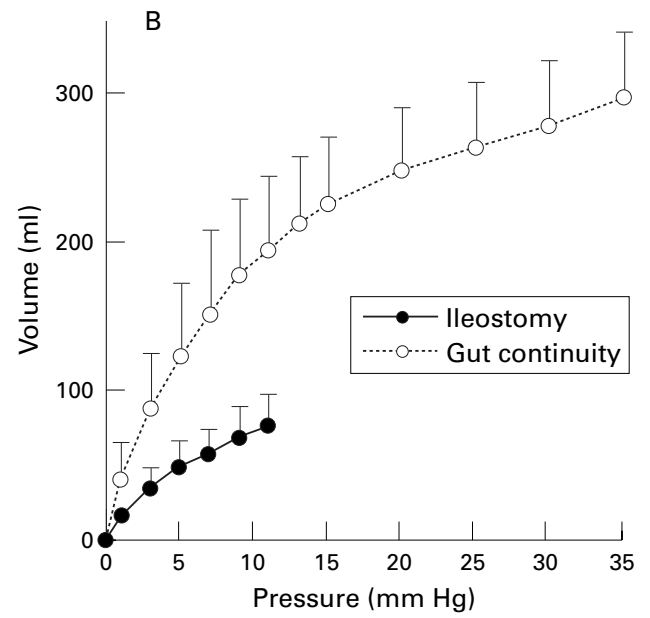

Figure 4 Pouch distensibility during volume-controlled $(A)$ and pressure-controlled $(B)$ distensions in patients with gut continuity or ileostomy. The patients with gut continuity tolerated the distensions up to the maximum tested volume of 300 $\mathrm{ml}$ and pressure of $35 \mathrm{~mm} \mathrm{Hg}$, whereas those with ileostomy interrupted the distensions from volumes $>90 \mathrm{ml}$ and pressures $>11 \mathrm{~mm} \mathrm{Hg}$. The pressure/volume ratio during volume-controlled distension was significantly lower in the patients with gut continuity than in those with ileostomy $(p=0.0001)$, and the slope of the volume/pressure relation during pressure-controlled distension was significantly steeper in the former than in the latter $(p=0.0001)$. Data are mean (SD) of pressures $(A)$ or volumes $(B)$ during distension. 


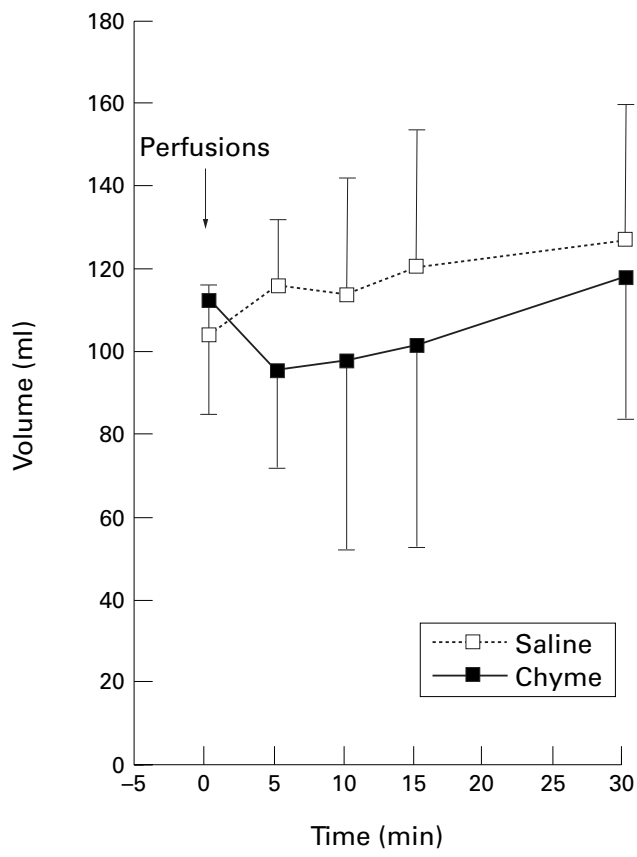

Figure 5 Effect of perfusions with chyme and saline on pouch tone in the patients with isolated pouches. The difference between baseline and the first five minute period after infusion with chyme was significant $(p=0.0044)$. The distending pressure was constant throughout the experiments. Data are mean (SD).

affect pouch volume (fig 5). The overall behaviour of pouch volume after the chyme and saline perfusions was not significantly different, but the difference between baseline and the first five minute period after infusion was significant $(p=0.0044)$. One phasic volume event was recorded in three patients during the five minutes preceding each perfusion; after both perfusions, phasic volume events were recorded in all of the patients, ranging from 2 to $10(5.67(3.50))$ and from 1 to $8(4.17$ (2.48)) after chyme and saline respectively.

\section{Discussion}

We observed increases in pouch tone and the frequency of phasic motor events after a meal in the patients with ileal pouches and gut continuity, but not in those whose ileal contents were diverted through an ileostomy; this suggests that the postprandial motor responses were related to the arrival of intraluminal contents in the pouch.

We verified that chyme could reach the pouch during the one postprandial hour of the experiment in which the ileal output of patients with ileostomy was collected. Immediately after the meal, the ileal output consisted of fluids and residues probably stored in the ileum after the previous meal $^{19}$; then the chyme appeared, and ileal output greatly increased. A delay in the progression of chyme can be expected in patients with gut continuity as a result of greater activation of the endocrine mechanisms regulating the ileal brake. ${ }^{20}$ However, this delay should not be too pronounced as the liquid component of a meal has been found to reach the pouch 30 minutes after its consumption, ${ }^{21}$ and most of our patients with gut continuity evacuated chyme at the end of the study.
To investigate whether the decrease in pouch volume observed after the meal may have been due to mechanical compression of the recording bag by intraluminal contents, or at least in part by an increase in pouch tone, we perfused the isolated pouches with chyme or an equal volume of saline. A decrease in pouch volume was observed only after the chyme perfusion, suggesting that the chyme stimulated a tonic contraction of the pouch. This result is in line with those of previous studies reporting that the contact of nutrients with gut mucosa stimulates gut tone. ${ }^{10}{ }^{22}$ It has been suggested that activation of chemoreceptors may be involved in these local responses, ${ }^{10}$ but the participation of endocrine mechanisms cannot be excluded. ${ }^{20}$ The results we obtained in isolated pouches suggest that the postprandial decrease in pouch volume observed in patients with gut continuity was at least partially attributable to an increase in pouch tone.

The frequency of phasic volume events also increased during the postprandial period in the patients with gut continuity. These events are possibly the equivalent of the high amplitude pressure waves measured by manometry, ${ }^{5}$ which have been shown to increase after a meal and to be triggered by the distension of the pouch to a threshold volume..$^{5}$ The increase in the frequency of phasic volume events in the postprandial period could be related to a distension caused by the ileal contents of a pouch, the threshold volume of which has been reduced by an increase in pouch tone. However, in line with previous observations showing that local nutrients increase phasic ileal motility, ${ }^{9}$ a direct effect of chyme on phasic volume events cannot be excluded. Finally, the increase in the number of pouch phasic contractions, which have previously been shown to be associated with defecation, ${ }^{6}$ may be related to the occurrence of bowel movements reported by our patients during the postprandial period.

The tone and distensibility of the isolated pouches were respectively greater and less than those of the pouches in continuity with the gut. Previous studies have shown that pouch volume is reduced in patients with ileostomy, ${ }^{3}$ and that pouch volume and distensibility increase during the first year after ileostomy closure. ${ }^{323}$ After ileostomy closure, it is conceivable that the muscular and connective tissue component of the pouch wall are progressively distended by intraluminal contents up to a maximum resting volume that depends on the type of pouch ${ }^{4}$ and pouch tone. Moreover, pouch volumes under fasting conditions linearly decreased in the patients with gut continuity but linearly increased in those with ileostomy. It is possible that the volume of ileal output may have influenced the resting volume of the pouch in continuity whereas, in patients with ileostomy, the small pressure exerted by the continuous isobaric distension was sufficient to counteract the hypertonic contraction of a pouch not previously distended by intraluminal contents.

Our patients with gut continuity tolerated distension volumes and pressures that were 
considered intolerable by those with ileostomy, who may be hypersensitive to visceral stimuli or the increased tone of the isolated pouch. Pharmacologically induced changes in gut tone have been shown to cause changes in gut distensibility and perception threshold levels during distension. ${ }^{24}$ According to these studies, the small volume and increased tone of isolated pouches may explain why the patients were hypersensitive to distension. Moreover, in line with the results of studies in which psychosensory stimulation has been reported to increase colonic sensations during distension, ${ }^{25}$ it is possible that the potentially stressful shorter time interval from surgery in the patients with ileostomy may have influenced their hypersensitivity to distension.

In conclusion, the tone and frequency of phasic motor events increased during the postprandial period in the ileal pouches that were in continuity with the gut, these effects being at least partially regulated by the local effect of intraluminal contents. The increase in pouch tone and the frequency of phasic volume events induced by the meal may favour defecation during the postprandial period.

The authors are grateful to the Associazione Amici della Gastroenterologia del Padiglione Granelli for financial support.

1 Parks AG, Nicholls RJ, Belliveau P. Proctocolectomy with ileal reservoir and anal anastomosis. $\mathrm{Br} \quad \mathcal{f}$ Surg 1980;67:533-8.

2 McIntyre PB, Pemberton JH, Wolff BG, et al. Comparing functional results one year and ten years after ileal pouchfunctional results one year and ten years after ileal pouchRectum 1994:37:303-7.

3 Oresland T, Fasth S, Nordgren S, et al. Pouch size: the important functional determinant after restorative proctocolectomy. Br F Surg 1990;77:265-9.

4 Nasmyth DG, Johnston D, Godwin PGR, et al. Factors influencing bowel function after ileal pouch-anal anastomosis. Br F Surg 1986;73:469-73.

5 O'Connell PR, Pemberton JH, Brown ML, et al. Determinants of stool frequency after ileal pouch-anal anastomosis. Am $\mathcal{F}$ Surg 1987;153:157-64.

6 Stryker SJ, Phillips SF, Dozois RR, et al. Anal and neorectal function after ileal pouch-anal anastomosis. Ann Surg 1986;203:55-61.
7 Ferrara A, Pemberton JH, Grotz RL, et al. Motor determinants of incontinence after ileal pouch-anal anastodeterminants of incontinence after
mosis. Br F Surg 1994;81:285-8.

8 Coffin B, Lémann M, Flourié B, et al. Ileal tone in humans: effects of locoregional distentions and eating. Am f Physiol 1994;267:G569-74.

9 Fich A, Phillips SF, Neri M, et al. Regulation of postprandial motility in the canine ileum. Am f Physiol 1990;259:G76774 .

10 Coffin B, Lémann M, Flourié B, et al. Local regulation of ileal tone in healthy humans. Am f Physiol 1997;272:G14753.

11 Hallgren T, Oresland T, Cantor P, et al. Intestinal intraluminal continuity is a prerequisite for the distal bowel motility response to feeding. Scand F Gastroenterol 1995;30:554-61.

12 Azpiroz F, Malagelada JR. Physiological variations in canine gastric tone measured by an electronic barostat. Am $\mathcal{F}$ Physiol 1985;248:G229-37.

13 Von Der Ohe MR, Hanson RB, Camilleri M. Comparison of simultaneous recordings of human colonic contractions by manometry and barostat. Neurogastroenterol Motil 1994; 6:213-22.

14 Sandborn WJ, Tremaine WJ, Batts KP, et al. Pouchitis after ileal pouch-anal anastomosis: a pouchitis disease activity index. Mayo Clin Proc 1994;69:409-15.

15 Bell AM, Pemberton JH, Hanson RB, et al. Variations in muscle tone of the human rectum: recordings with an electromechanical barostat. Am f Physiol 1991;260:G17-25.

16 Kirk P. Split-plot factorial design: design with grouptreatment confounding. In: Kirk P, ed. Experimental design. Procedures for the behavioral sciences. Belmont: Brooks Cole Procedures for the behavioral sciences. B

17 Kirk P. Orthogonal polynomials. In: Kirk P, ed. Experimental design. Procedures for the behavioral sciences. Belmont: Brooks Cole Publishing Company, 1982:149-61.

18 Huynh H, Feldt LS. Estimation of the Box correction for degrees of freedom from sample data in randomized block and split-plot designs. Fournal of Educational Statistics 1976; 1:69-82.

19 Camilleri M, Colemont LJ, Phillips SF, et al. Human gastric emptying and colonic filling of solids characterized by a new method. Am 7 Physiol 1989;257:G284-90.

20 Soper NJ, Chapman NJ, Kelly KA, et al. The "ileal brake" after ileal pouch-anal anastomosis. Gastroenterology 1990; 98:111-16.

21 Goldberg PA, Kamm MA, Nicholls RJ, et al. Contribution of gastrointestinal transit and pouch characteristics in determining pouch function. Gut 1997;40:790-3.

22 Basilisco G, Phillips SF, Cullen JJ, et al. Tonic responses of canine proximal colon: effects of eating, nutrients, and simulated diarrhea. Am F Physiol 1995;268:G95-101.

23 Sagar PM, Holdsworth PJ, Johnston D. Correlation between laboratory findings and clinical outcome after restorative proctocolectomy: serial studies in 20 patients with end-toend pouch-anal anastomosis. Br f Surg 1991;78:67-70.

24 Notivol R, Coffin B, Azpiroz F, et al. Gastric tone determines the sensitivity of the stomach to distention. Gastroenterology 1995;108:330-6.

25 Ford MJ, Camilleri M, Zinsmeister AR, et al. Psychosensory modulation of colonic sensation in the human transverse and sigmoid colon. Gastroenterology 1995;109:1772-80. 